
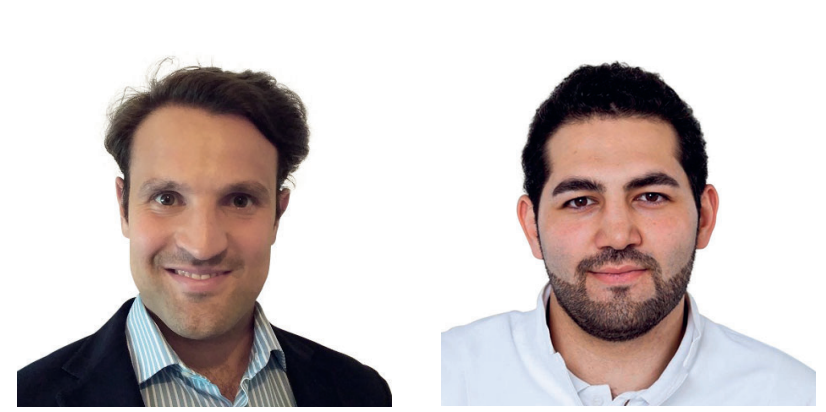

\title{
Trockenes Auge und Diabetes mellitus: Selbsteinschätzung und Lebensqualität
}

\author{
Emiliano Di Carlo Fadi Raslan
}

Augenklinik, Städtisches Klinikum Karlsruhe, Karlsruhe, Deutschland

Zusammenfassung zu Yazdani-Ibn-Taz MK, Han MM, Jonuscheit S, et al.: Patient-reported severity of dry eye and quality of life in diabetes. Clin Ophthalmol. 2019;13:217-224.

\section{Schlüsselwörter}

dry eye $\cdot$ ocular surface $\cdot$ retinopathy $\cdot$ symptoms

\section{Zusammenfassung}

Ziel der Studie war es, den Zusammenhang zwischen den subjektiv empfundenen Beschwerden des Trockenen Auges, der Lebensqualität, dem Vorliegen einer diabetischen Retinopathie (DR) und der Krankheitsdauer bei Patienten mit Diabetes mellitus Typ 1 (DM1) und Typ 2 (DM2) zu ermitteln. Dazu beantworteten 152 Patienten (110 Diabetiker und 42 ohne Diabetes in der Kontrollgruppe) je zwei Fragebögen (Ocular Surface Disease Index, Dry Eye-realted Quality of Life Score).
Von den Diabetes-Patienten berichteten 44\% von Symptomen des Trockenen Auges, wobei der Anteil unter DM2-Patienten höher war (55\% verglichen mit 27\% bei DM1-Patienten, $p=0,001$ ). In der Kontrollgruppe berichtete 29\% von Symptomen des Trockenen Auges. Die Schwere der Symptome ging mit einer sigknifikanten Abnahme der Lebensqualität sowohl bei DM1 als auch bei DM2 einher (DM1: $r=0,609, p=0,036$; DM2: $r=0,417, p=0,011$ ). Unabhängig von einem Vorliegen einer DR trat das Trockene Auge häufiger bei DM2 als bei DM1 auf (mit DR: $p=0,011$; ohne DR: $p=0,018)$.

C 2019 S. Karger GmbH, Freiburg 


\section{Transfer in die Praxis}

\section{Hintergrund}

Diabetes mellitus ist eine der weltweit führenden Ursachen für Morbidität und Mortalität [1]. Das mit der Erkrankung oft einhergehende Trockene Auge (DED, Dry eye disease) hat einen signifikanten Einfluss auf die Lebensqualität der Patienten (QoL, Quality of Life) durch körperliche, soziale und/oder psychologisch negative Auswirkungen [2]. Des Weiteren kommt es zu Einschränkung der Produktivität am Arbeitsplatz. Ziel der Studie von Yazdani-Ibn-Taz et al. war es, zunächst das Ausmaß der Schwere des Trockenen Auges und die Auswirkungen auf die Lebensqualität bei Typ-1- (DM1) und Typ-2 (DM2)-Diabetikern im Vergleich zu gesunden Patienten herzustellen. Das zweite Ziel war es, die Assoziation von DED und QoL mit Alter, Geschlecht, Dauer der an Diabetes mellitus erkrankten Personen, der Diabetischen Retinopathie (DR), Makulopathie und Neuropathie und des HbA1c-Wertes zu finden.

\section{Ergebnisse der Studie}

Es handelt sich hier um eine retrospektive Studie mit insgesamt 152 Probanden (davon 110 mit Diabetes diagnostiziert und 42 gesunde Studienteilnehmer), die mit Hilfe zwei verschiedener Fragebögen durchgeführt wurde: OSDI (Ocular Surface disease Index), basierend auf subjektiven Symptomen, sowie DEQS (Dry Eye related Quality of Life Score). Laut dem OSDI-Fragebogen berichteten 44\% der 110 Diabetiker über Symptome des Trockenen Auges. Die Mehrheit der durch Symptome geplagten Patienten befand sich jedoch in der DM2-Gruppe. Sowohl bei DM2 als auch bei DM1 waren die Beschwerden signifikant höher als in der Kontrollgruppe. Bei ausgeprägten Symptomen war die gemessene QoL bei Patienten mit DM2 niedriger als bei DM1. Es konnte eine positive Korrelation zwischen DEQS, einem erhöhten HbA1c-Wert und der QoL bei DM2 festgestellt werden.

Das Auftreten von trockenen Augen war signifikant höher bei DM2 mit DR (63\%) als bei DM1 mit DR (28\%).

\section{Kritik}

Patienten mit Diabetes leiden häufiger an trockenen Augen als diejenigen ohne Diabetes. Die Studie zeigt den Zusammenhang zwischen trockenen Augen und den negativen Auswirkungen auf QoL, von der hauptsächlich die Typ-2-Diabetiker beeinflusst sind [3], unabhängig von anderen demographischen Daten [4]. Darüber hinaus bewiesen frühere Studien keine statistisch relevanten Unterschiede zwischen den beiden Diabetesformen. Dieser Aspekt ist kontrovers: Die Autoren der Studie vermuten, dass die Ursache des Unterschiedes der Symptome zwischen DM1 und DM2 von verschiedenen pathophysiologischen Prozessen abhängen könnten. Interessant wäre es, die Anwesenheit von pro-inflammatorischen
Zytokinen im Tränenfilm von Diabetikern zu messen und diese mit den Werten der subjektiven Fragebögen zu korrelieren.

Des Weiteren ließ sich keine nennenswerte Differenz bezüglich der Symptomatik der trockenen Augen zwischen Patienten mit DM1 und den gesunden Probanden feststellen [5]. In zukünftigen Studien sollte das Verhältnis zwischen DR und QoL durch die Integration anderer Faktoren wie Einnahme von Medikamenten, Art der Arbeit, Kontaktlinsen und Lasertherapie berücksichtigt werden.

\section{Fazit für die Praxis}

Die Anzahl der an Diabetes erkrankten Menschen steigt jährlich weltweit und stellt Ärzte stetig vor neue Herausforderungen. Die hier vorgestellte Studie liefert wichtige Information über die Rolle, die eine sorgfältige Diagnostik des Trockenen Auges bei Patienten mit Diabetes mellitus spielt.

Meines Erachtens können die hier vorgestellten beiden Fragebögen (DEQS und OSDI) in der klinischen Praxis als Referenzparameter regelmäßig verwendet werden. Das Ziel ist es, eine richtige und gezielte Therapie anzusetzen, um immer wieder auftretende Beschwerden, wie z.B. Sandkorngefühl und Epiphora, lindern zu können.

Eine sofortige Einleitung der Therapie mit Tränenersatzmittel und Lidhygiene ist schon zu Beginn der Beschwerden zu empfehlen, um so einer Einschränkung der Lebensqualität entgegenwirken zu können.

\section{Disclosure statement}

Hiermit erkläre ich, dass keine Interesskonflikt in Bezug auf den vorliegenden Kommentra bestehen.

\section{Literatur}

1 Papatheodorou K, Banach M, Edmonds M, et al.: Complications of diabetes. J Diabetes Res. 2015;2015:189525.

2 van Tilborg MM, Murphy PJ, Evans KS: Impact of dry eye symptoms and daily activities in a modern office. Optom Vis Sci. 2017;94: 688-693.

3 Zou X, Lu L, Xu Y, et al.: Prevalence and clinical characteristics of dry eye disease in community-based type 2 diabetic patients: the Beixinjing eye study. BMC Ophthalmol. 2018;18:117

4 Achtsidis V, Eleftheriadou I, Kozanidou E, et al.: Dry eye syndrome in subjects with diabetes and association with neuropathy. Diabetes Care. 2014; 37:e210-1.

5 Hashemi H, Khabazkhoob M, Kheirkhah A, et al.: Prevalence of dry eye syndrome in an adult population. Clin Exp Ophthalmol. 2014;42: 242-248.

Kontaktadresse: Dr. Emiliano Di Carlo, Augenklinik, Städtisches Klinikum Karlsruhe, Moltkestraße 90, 76133 Karlsruhe, Deutschland, emi.dicarlo@hotmail.it 